\title{
物理化学课程在线教学实践与效果分析
}

任冬梅 ${ }^{*}$, 王力霞, 赵岩, 夏云生

渤海大学化学与材料工程学院, 辽宁锦州 121013

摘要: 介绍了渤海大学的物理化学课程在钉钉平台进行线上授课的主要举措及经验。通过整个学期的实践可知, 所构 建的 “简单-详细一归纳” 的模块化教学模式有利于不同自学能力的学生与不同层次的教学相匹配, 我问你答式的视频 归纳课可以充分调动学生的学习积极性, 有利于教学相长和师生互动的完美实现。在细致透明的教学管理、形影相随 的过程考核的双重保障下, 整个学期的课程总目标达成度达 $80 \%$ 以上, 分目标达成度均在 $60 \%$ 以上, 这为相关教学提供 了借鉴。

关键词: 在线教学; 物理化学; 钉钉平台; 模块教学

中图分类号: G64; O64

\section{Online Teaching Practice of Physical Chemistry Course and Effect Analysis}

\author{
Dongmei Ren *, Lixia Wang, Yan Zhao, Yunsheng Xia \\ College of Chemistry and Materials Engineering, Bohai University, Jinzhou 121013, Liaoning Province, China.
}

\begin{abstract}
The main measures and experiences of online physical chemistry teaching through Ding Talk platform in Bohai University was introduced. Through the practice of the whole semester, it can be seen that the modular teaching mode of "simplicity-detail-induction" constructed by physical chemistry teaching team is conducive to match students with different self-learning abilities with different levels of teaching. The question-and-answer video induction class can fully arouse students' learning enthusiasm, which is favorable for the perfect realization of teaching and teacherstudent interaction. With the double guarantee of meticulous and transparent teaching management and the accompanying process assessment, the overall goal achievement degree of the whole semester reached beyond $80 \%$, and the sub-goal achievement degree reached over $60 \%$, which provided reference for relevant teaching.
\end{abstract}

Key Words: Online teaching; Physical chemistry; Ding Talk; Modular teaching

物理化学作为化学学科的一个分支, 是化学专业及与化学密切相关专业的一门主干基础课。由

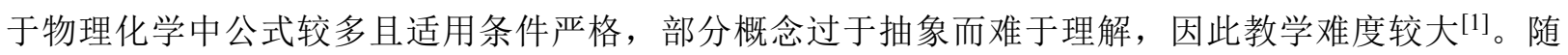
着高校教育的全面改革, OBE (Outcomes-based Education)教学理念 ${ }^{[2]}$ 及微课、慕课等线上教学形 式 ${ }^{[3,4]}$ 均对传统教学产生了积极而深远的影响。如何充分利用网络资源, 激发学生的学习兴趣和课堂 参与积极性, 发挥学生自主学习的潜能, 保证学生掌握物理化学课程的专业技能, 达到课程目标, 是高校物理化学教师共同面临的重要问题。

收稿: 2020-08-07; 录用: 2020-09-01; 网络发表: 2020-09-21

“通讯作者, Email: rendm582@163.com

基金资助：渤海大学校级教改项目(BDJGCY2019117) 
疫情期间, 众多网络教学平台在高校线上教学过程中发挥了重要作用, 每位教师均对线上教学 设计、教学过程、教学效果以及教学评价等方面进行了有益的探析 ${ }^{[5-8]}$ 。为适应我校学情, 我们综合 比较了各类平台, 最终我校的物理化学课程确定选用操作便捷、互动性好、稳定流畅的 “钉钉” 作 为主要的教学平台, 辅以 “超星” 和 “问卷星”, 对物理化学的线上教学方法及过程进行了探索及实 践, 以期为相关教学的开展提供借鉴。

\section{1 物理化学课程线上教学实践}

本学期的物理化学课程为化学热力学部分, 教材为南京大学傅献彩编写的《物理化学》(第 5 版)上 册。内容包含绪论、气体、热力学定律、多组分热力学、相平衡及化学平衡, 上课 17 周, 共计 68 学时。 物理化学课程线上教学与传统教学方式的相同之处在于, 整个教学过程均分成管理、资源及教 学三个部分。“管理” 主要是实现两个方面的管理, 一是授课内容的总体安排及简介, 使学生能够 及时了解课程的进度和具体要求, 二是对选课学生学习日常状态进行管理。“资源” 主要是指教学 中为学生提供的基本资料和拓展资料, 学生可随时在线观看, 下载学习。“教学” 则是指具体的课 堂教学, 通过课程讲授、课程作业和在线测试来完成学习者的自我学习评价。线上教学与传统教学 方式的不同之处在于网络资源丰富、学习者不受时空的限制, 但其学习的自觉性和学习状态需要教 师监督与激励。因此线上教学中各个部分的完成方式与传统教学方式相比有所不同, 尤其是教学管 理及课程讲授过程。

\section{1 管理细致透明}

学生自学能力的培养一直是教学的重点, 也是教学的难点。线上教学过程中, 为提升学生自学 能力, 教学计划管理、教学组织管理等基本环节需加以细化统筹。

教学计划管理的细致透明是指制定一份目标任务清晰、学习计划详细、进度安排合理的教学计 划表, 通过钉钉平台的 “家校通知” 或 “新建任务” 功能展示给学生。教学计划表包含三个部分, 月度计划表, 章节计划表, 课前计划表。月度计划表以索引方式安排每月课程的总体进度; 章节计 划表指当月的每次课程完成的章节学习内容、相应知识点视频资源、学习时间等给出建议; 课前计 划表指下次课需要完成的具体目标, 通常通过 “DING一下” 功能在上课的前一天提醒学生。这样, 可以使学生有规划、有步骤自我管理, 也方便学生带着问题去学习。

学生学习的自我规划与自觉管理是变被动学习为主动学习的关键, 而使学习成为习惯的过程中 教师适时的督学作用不可缺少。及时要求学生签到、签退, 通过平台记录, 关注学生的学习时长是 督学过程中必要的管理手段。由于钉钉平台的签到功能是无法自动签到或无法补签, 而签到地址亦 是无法更改的, 这能在一定程度上对学生起到督促作用。为提高互动性还可以通过 “家校本” 方式 实现图片签到功能, 学生签到的积极性也较高。整个学期的教学实践中, 通过打卡方式签到的学生 参与度可在 $95 \%$ 以上, 视频或照片打卡的参与度可达 $100 \%$, 可见学生更易接受互动式网络教学, 并 在督学的过程中表现出了良好的学习自觉性。

\section{2 教学分层模块}

线上教学虽然缺少了师生面对面的交流, 但丰富多样的网络学习资源、学习时间不必受课时限 制等优势充分尊重学习者的学习主体地位。然而, 学习者的自学能力有着较大的差异, 因此在线教 育势必要做到讲授有层次、与学生接受相匹配才能发挥网络教学的巨大扩张力。经半年的教学实践, 物理化学课程逐渐形成了 “简单-详细-归纳” 的分层模块教学模式(如图 1 所示)。

(1) 简单教学模块主要利用 “超星” 平台教学资源。该平台上的大连理工大学物理化学上册全部 教学内容的教学视频仅 $9.6 \mathrm{~h}$, 因此可以用做预习性讲解。新单元开始即要求学生利用 1-2 课时在平 台讲解下进行全单元自主预习。经过讲解, 理解能力较强的学生可以掌握大部分的内容。理解能力 较弱的学生也能做到心中有数, 哪些是自己根本不懂的内容, 哪些是自己比较疑惑的地方, 从而掌 握学习的主动权。简单教学模块课时约占总课时的 $1 / 5$ 。 


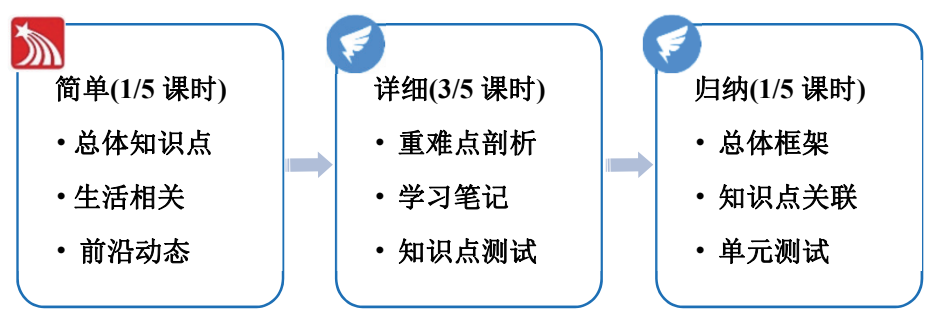

图 1 线上物理化学课程的模块式教学

(2) 详细教学模块是在简单教学模块之后进行, 这部分的课时占总课时的 $3 / 5$ 。该模块是根据本 校学生学情, 以录播或直播的方式详细并有针对性地讲解知识点。提出适应本校教学的学习要求, 指出重点和难点, 强调各部分学习的意义及相互联系。需要指出的是, 直播教学应尽量选择钉钉视 频会议(或在线课程)形式, 这有利于设计问题, 在学生掌握课堂内容的基础上, 进行课堂讨论, 即时 沟通, 提高线上教学的互动性。在这部分教学中, 目标须清楚地表述, 切中课程特定具体的要求, 不可模棱两可。教学过程中可以采用手写笔演示功能, 以提高学生思维的跟随度, 并有利于学生课 后针对上课过程中所学知识进行总结, 记录课堂笔记。直播或录播教学完毕, 利用钉钉平台的 “家 校本”功能设置作业，明确作业完成的截止时间、平时考核的截止时间等。

该模块可让自学能力强的学生全面掌握本章的知识点, 彻底解决在简单模块中遗留的问题, 构 建更完善的知识体系, 融会贯通。也可让自学能力较弱的学生系统掌握基础知识, 领会本单元的重 点。此外, 同步作业批改可以通过平台的语音批改功能进行有针对性的讲解, 把课堂未详细讲解的 知识进一步完善, 不留死角。

(3) 知识点归纳总结模块是教学中极为重要的一环。该模块的课时虽然仅占总课时的 $1 / 5$, 但这 部分课程的完成必须通过视频直播课完成。归纳总结课时重点是要帮助学生进一步理清概念, 搭建 知识网络, 着重突出知识点之间的逻辑关系及整体知识结构。就重要理论、重点、难点进行分析总 结, 安排课堂讨论, 通过我问你答的方式解决学习中的共性问题。

该模块教学中, 学生还可以随时对不理解且感兴趣的内容进行提问, 教师在解答过程中不仅可 以进行扩展性的补充和解释, 而且可以通过互动再次了解学生学习中存在的问题, 有针对性地加以 指导, 并有针对性地布置课后作业巩固相关知识点。通过对知识点的系统整理和综合, 辅以广泛深入 而有针对性的讨论和交流, 将学生的疑惑再次减少, 形成教学活动中最有特色、最有活力的学习形式。

图 2 给出了本学期物理化学课程 “相律” 部分的模块式教学实施过程。超星平台将该部分课程 分成 “独立组分数” “相律” 及 “相律推导” 三个部分, 讲解始于 $C=S-R-R$ ”的计算, 时长共约 $32 \mathrm{~min}$ 。经过该部分学习, 学生基本上可以掌握研究系统的独立组分数及自由度的计算方法。基础 较好的学生对于相及相数的理解基本没有障碍, 但对于 $R^{\prime}$ 的理解及运用能力较差, 而基础较弱的同 学对于 $\Phi$ 的确定已经比较模糊, 仅能机械记忆 $C$ 及 $f$ 的两个计算公式。

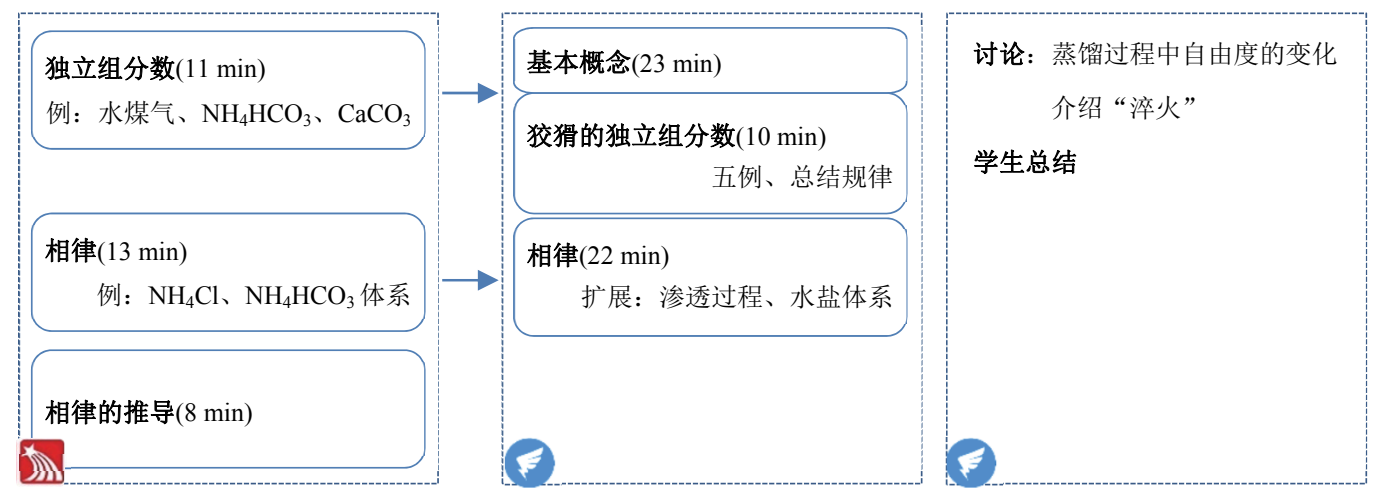

图 2 相律的模块式教学实施过程 
结合随堂提问的反馈及知识重难点要求, 我们在钉钉平台上将独立组分数及相律部分扩展, 时 长共约 $55 \mathrm{~min}$ 。独立组分数扩展成 “基本概念” 和 “狡猾的独立组分数” 两部分：基本概念详细介 绍关于相的概念、关于物质的概念及自由度的概念; 狡猾的独立组分数中以 HCN 水溶液等系统为 例, 通过不同的计算方法均得到相同的 $C$, 从而总结出计算规律为考虑问题的角度不同, 物种数可 以不同, 但组分数唯一。相律部分的扩展主要体现在应用系统扩展, 添加了常见渗透系统及水盐系 统, 加强学生对相律的灵活运用能力。由于简单模块已经将相律推导部分介绍得比较清楚, 则不予 以扩展。经过对模块详细的讲解, 所有学生对相律部分的知识体系已经能达到清晰明了, 基础好的 学生已经可以自行解决相对难一些的习题了。

最后, 通过作业反馈, 在钉钉平台上以学生讨论、抢答计分等方式对即将要接触到的蒸馏过程 和合金系统进行自由度的计算, 由学生自主总结知识点, 并指出各公式的使用注意事项, 则学生能 完全掌握该部分知识, 达到教学目的。

综上可见, 经过三次不同层次模块教学, 学生学习积极性可以充分调动, 并且可以满足学生的 个性化需求, 便于不同自学能力的学生与不同层次的教学相匹配, 有利于教学相长和师生互动的完 美实现。

\section{3 评测形影相随}

为适应线上教学过程, 课程考核方式也将相应地有所改变。本学期物理化学课程考核方式中期 末理论考试占比降低, 为 $40 \%$, 平时过程考核比例提高至 $50 \%$, 出勤以课堂表现代替, 占比为 $10 \%$ 。

平时考核的成绩来源主要是布置的作业以及章节后的测试。作业的布置及批改均由钉钉平台完 成, 而网络平台 “问卷星” 能够提供方便的在线测试功能。每单元结束, 本课程均设计了考查基本 知识点的测试性习题, 要求学生通过手机扫描二维码, 限时、独立完成测试过程。问卷星平台可以 详实获得学生的总体学习情况(题目正确率等)和学生的个别完成情况。这将有利于依据题目的正确 率调整讨论或评讲方式, 及时调节教学进度。此外, 利用网络平台保证了每个学生都能积极参与完成 学习任务, 并且教师能够在无需收、阅卷的情况下快捷掌握学生的学习情况, 提高教学效率。整个 学期, 学生的平时成绩平均为 45.7 分 (总分 50 分), 参与度为 $100 \%$, 表明学生是乐于并积极参与在 线学习的。由此可见, 降低总结性考试, 增加过程性考核, 使考核如影随形, 有利于充分激励学生 平时自主学习物理化学课程的热情, 亦是教师督学、导学的重要手段。

\section{2 课程目标达成度分析}

目前我校正在推行 OBE 的教学模式, 课程目标达成度评价系统是 OBE 教学改革项目之一。物 理化学课程教学目标体现在知识(目标 1)、技能(目标 2)及素养(目标 3)三个方面, 目前成绩考核的来 源分别是课堂表现(10\%), 平时考核 (50\%)以及期末考试(40\%)。平时考核中的 50 分中有 30 分对应课 堂目标 1 层次, 20 分对应课堂目标 2 层次; 期末考试中 24 分对应课堂目标 1 的难度, 课堂目标 2 及课堂目标 3 的难度均为 8 分; 课堂讨论的 10 分中有 4 分来自于基本知识的讨论(课程目标 1), 其 余 6 分对应综合能力(课程目标 2 和 3)。

图 3 给出了线上及线下物理化学课程目标达成度比较。本次线上教学参与考试的 2018 级学生 共计 141 人, 有效试卷 141 份。上一届参与线下教学的 2017 级学生计 135 人, 有效试卷为 135 份。 由图 3 可知, 与线下教学相比, 线上教学的总体目标达成度良好 $(86 \% 、 83 \%)$, 且课程目标 1 、课程 目标 2 目标达成度均在 $80 \%$ 以上, 说明选课学生对于课程的基础知识和原理掌握较好, 已经具备运 用物理化学方法解决问题的能力, 即运用化学热力学原理和方法分析问题和解决问题的能力。但线 上教学的两个班的目标 3 达成度仅为 $68 \%$ 和 $61 \%$, 低于线下教学 $(73 \%)$, 可见线上教学过程应加强 综合性知识的引导与练习, 即归纳模块的强度应予以适当增加。 

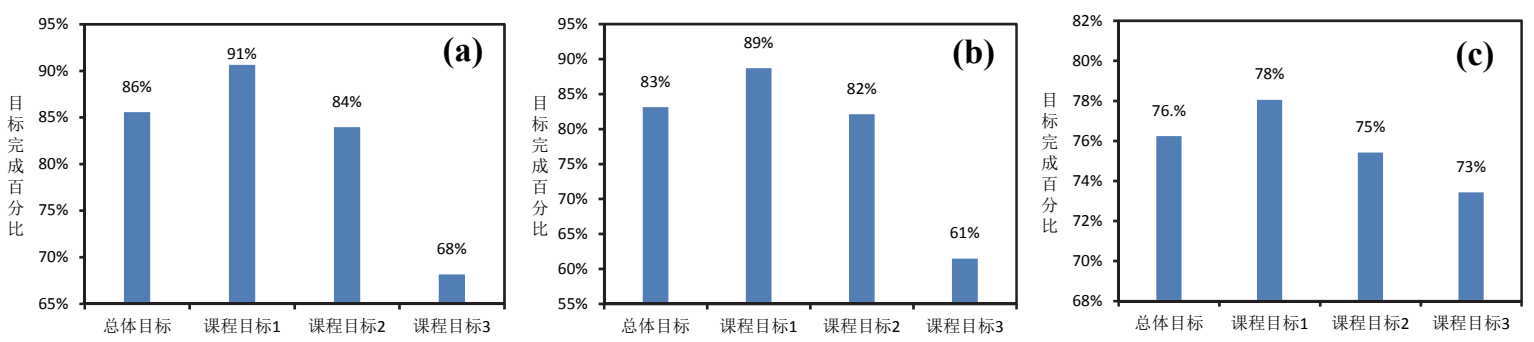

图 3 物理化学课程目标达成度分析

(a) 线上教学 $1 、 2$ 班; (b) 线上教学 $5 、 6$ 班; (c) 线下教学

\section{3 结语}

以钉钉为授课的主要平台, 进行了 “简单-详细-归纳” 三模块式线上物理化学的教学实践。结 果表明:

(1) 线上教学资源丰富, 但学习者的自我管理能力参差不齐, 透明精细化的教学管理有利于发 挥教师的监督及激励作用, 而过程性考核比例的增大亦可促进学生学习的自觉性及主动性。

(2) “简单-详细-归纳” 的分层模块式教学可以使不同学习能力的学生都能够从在线教学中得 到知识和能力的提升, 从而达到教学目的, 完成培养目标。

（3）课程的总体目标达成度均可达 $80 \%$ 以上, 分目标达成度均在 $60 \%$ 以上, 表明选课学生对于 课程的基础知识和原理掌握较好, 已经具备运用物理化学方法解决问题的能力, 模块化的网络教学 是适当并合理的教学形式。

(4) 教师通过出镜的视频直播归纳总结知识点, 更便于学生构建完整的知识体系, 突出重点和 难点, 更好地把握知识链的关键环节, 为深入学习和将来的深造打下坚实的理论基础。同时也能让 教与学在轻松和谐的氛围下完成, 起到画龙点睛、知识升华的良好效果, 是在线教学中的重要一环。

[1] 彭昌军, 徐首红, 刚洪泽, 殷天翔, 胡军, 尚亚卓, 刘洪来, 黑恩成. 大学化学, 2021, 36 (8), 1.

[2] 宗丽娜, 王向鹏, 郑云香. 化学教育(中英文), 2020, 41 (14), 14.

[3] 杨小敏, 刘建平, 胡林, 夏坚. 化学教育(中英文), 2018, 39 (24), 20.

[4] 余晓云, 叶剑标. 南方农机, 2020, 51 (11), 175.

[5] 原弘, 李武客, 王俊, 贾法龙, 王峰云, 郭军, 罗竹, 欧阳述昕. 大学化学, 2021, in press. doi: 10.3866/PKU.DXHX202005052

[6] 王旭珍, 王新葵, 王新平, 石川, 任素贞, 田东旭, 陈冰冰. 大学化学, 2020, 35 (5), 205.

[7] 冯霞, 刘俊吉, 李松林, 孙艳, 朱荣娇, 朱莉娜, 陈丽, 王玉新, 张小涛. 大学化学, 2020, 35 (5), 92.

[8] 高啨辉, 貲孟婕, 孔繁博, 袁廷, 李晓宏, 祖莉莉, 李运超, 范楼珍. 大学化学, 2020, 35 (5), 81. 